Supporting Information

\title{
Largely Improved Battery Performance Using Micro-sized Silicon Skeleton Caged By Polypyrrole As Anode
}

Yingying Lv, Mingwei Shang, Xi Chen, Parisa Shabani Nezhad, Junjie Niu* Department of Materials Science and Engineering, University of WisconsinMilwaukee, Milwaukee, WI 53211, USA

*Email: niu@uwm.edu

\section{This file includes:}

Figures $\mathrm{S} 1$ to $\mathrm{S} 12$ with legends

Table S1 

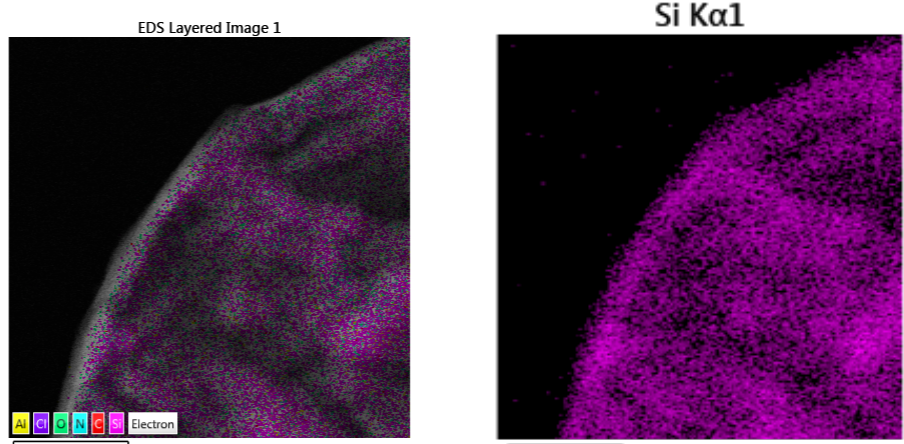

Al $\mathrm{K} \alpha 1$
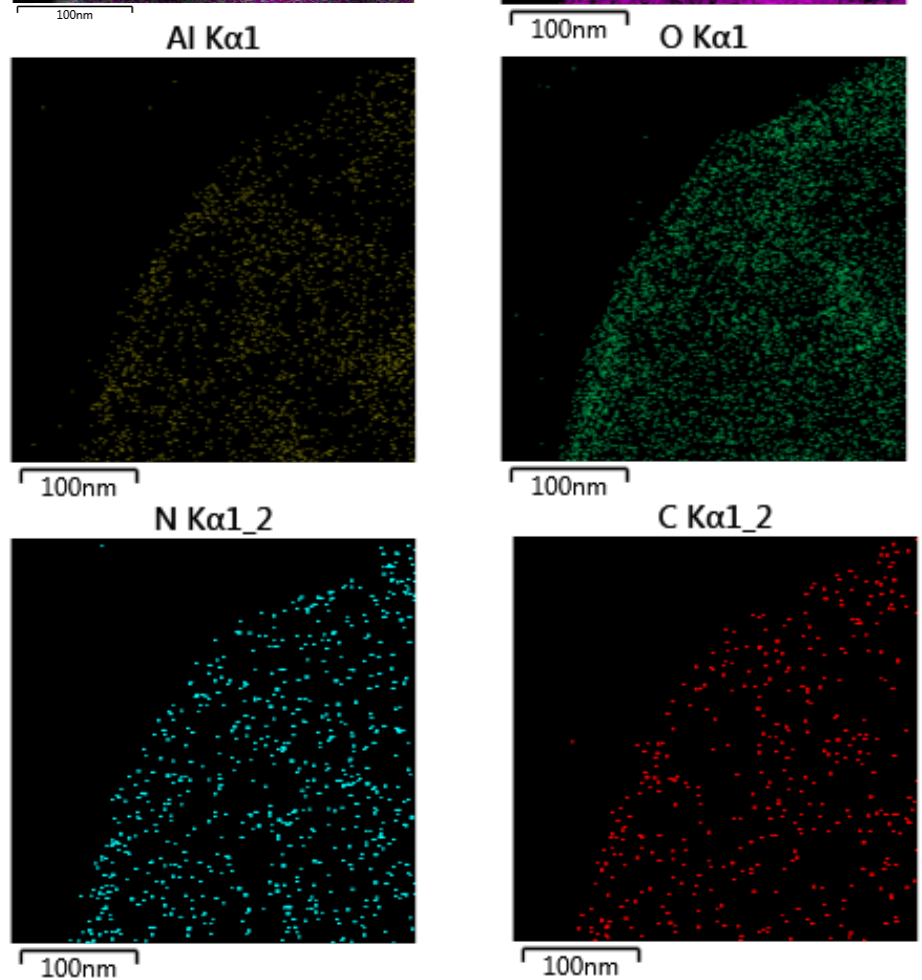

Figure S1. Elemental mapping of the $\mu$-Si cage by TEM energy dispersive X-ray spectroscopy. 

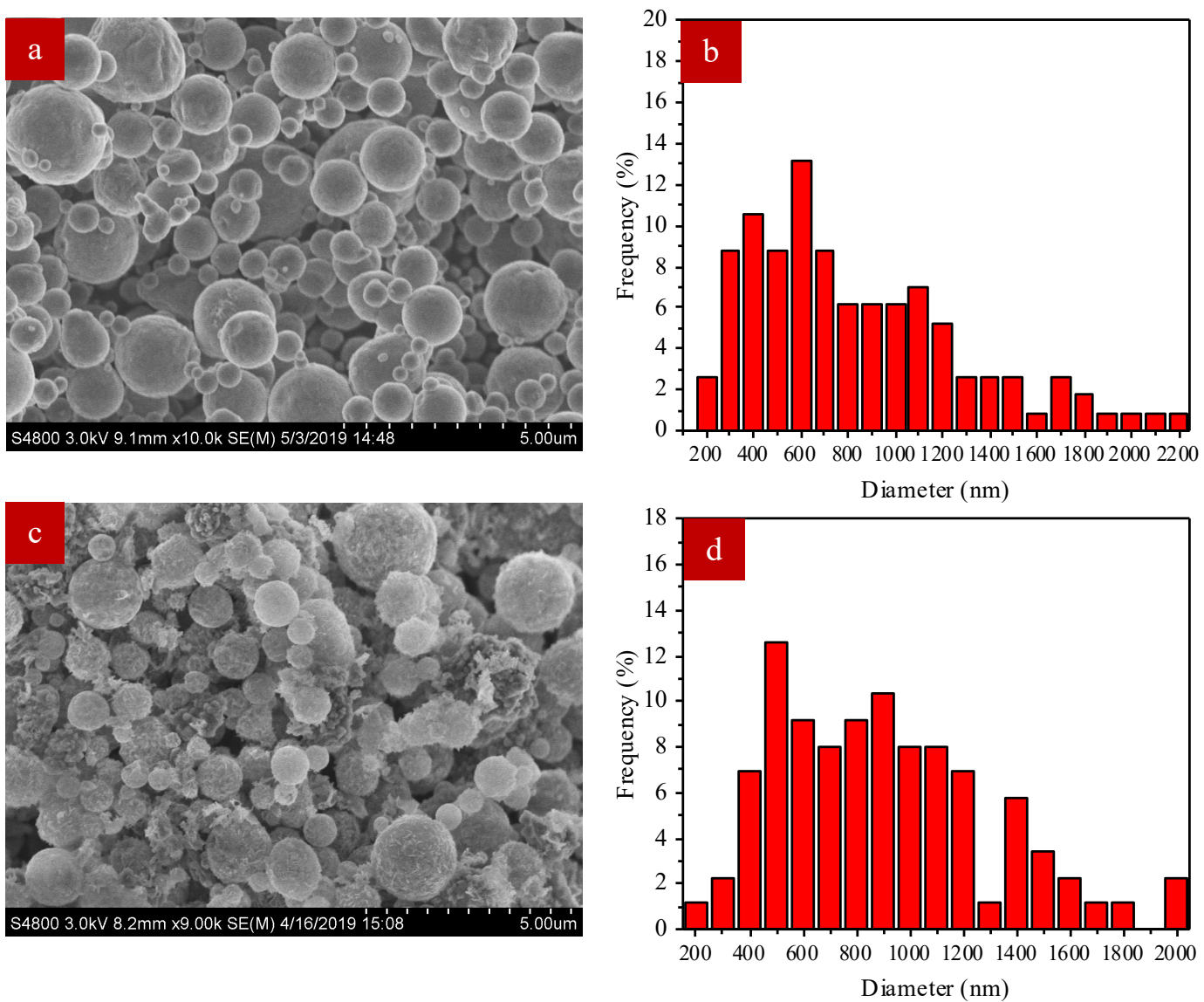

Figure S2. SEM images and corresponding size distribution of the AlSi alloy $(a, b)$ and $\mu$-Si cage $(c, d)$. 


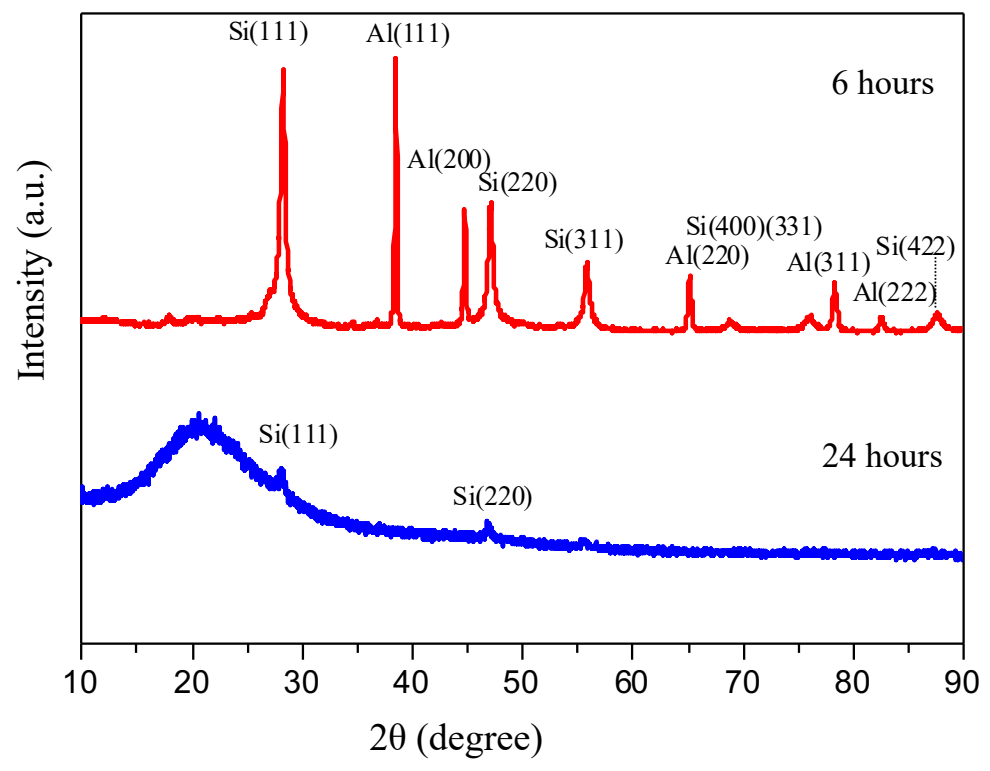

Figure S3. XRD curves of the AlSi alloy after direct etching with $6 \mathrm{M}$ $\mathrm{HCl}$ for 6 hours (red) and 24 hours (blue). 


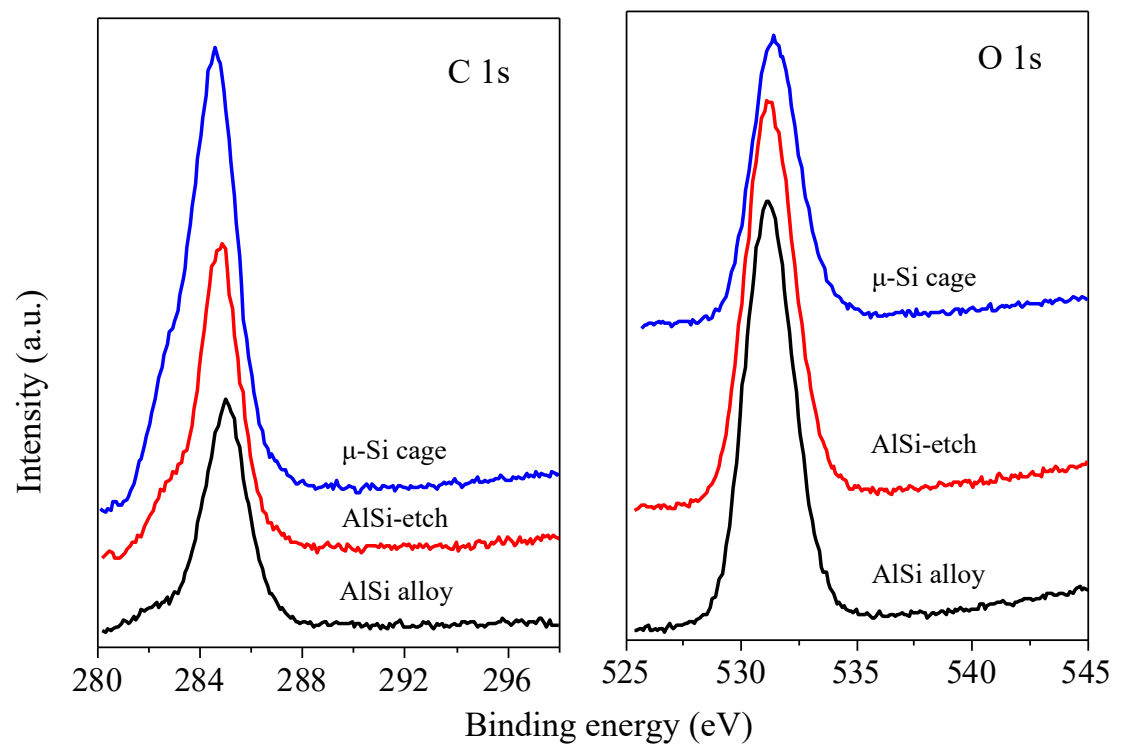

Figure S4. The C 1s and O 1s XPS peaks of pure AlSi alloy (black), AlSi alloy after direct etching (red) and $\mu$-Si cage (blue) . 


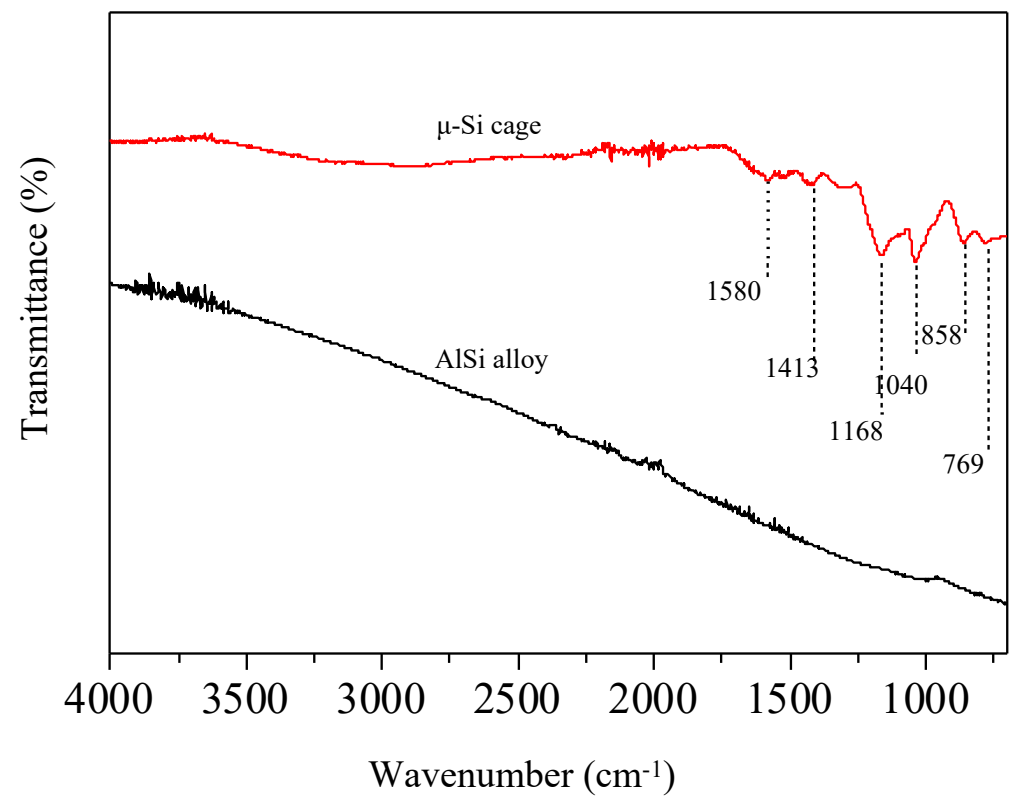

Figure S5. FTIR curves of pure AlSi alloy (black) and the $\mu-\mathrm{Si}$ cage (red). 

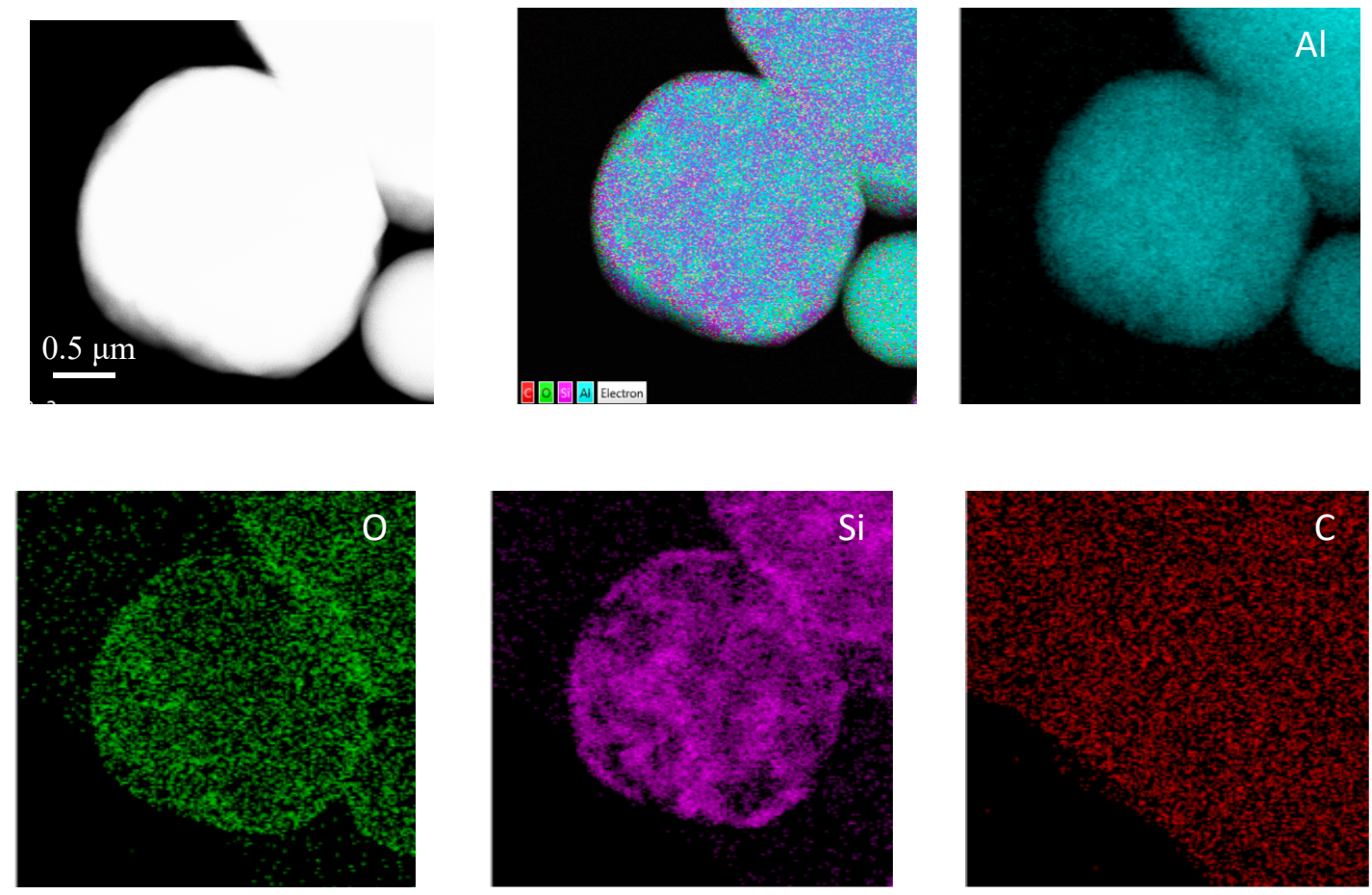

Figure S6. Scanning TEM (STEM) image and the corresponding elemental mappings of the original AlSi alloy. 


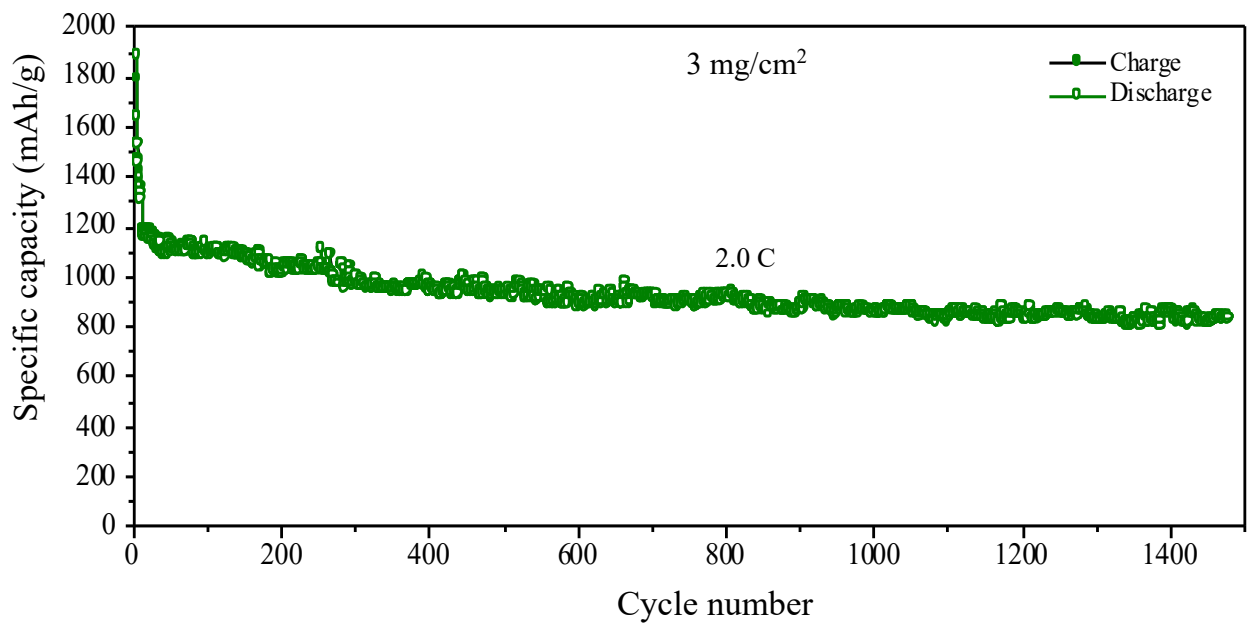

Figure S7. Cycling performance of the battery with the $\mu$-Si cage as anode with a loading of $3 \mathrm{mg} / \mathrm{cm}^{2}$ at $2.0 \mathrm{C}$. The specific capacity was calculated based on the pure silicon inside $\mu$-Si cage. 


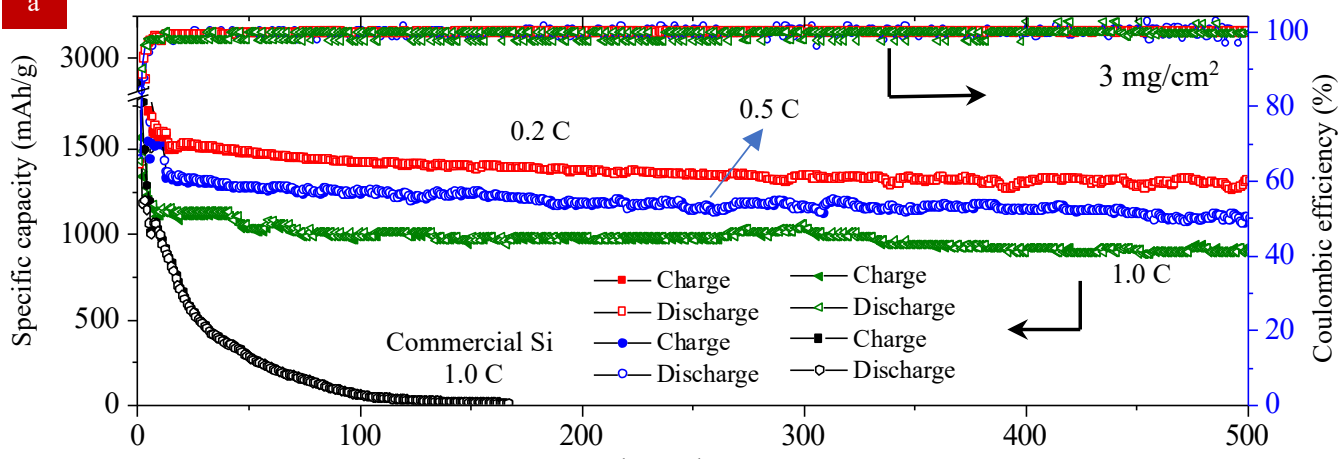

Cycle number

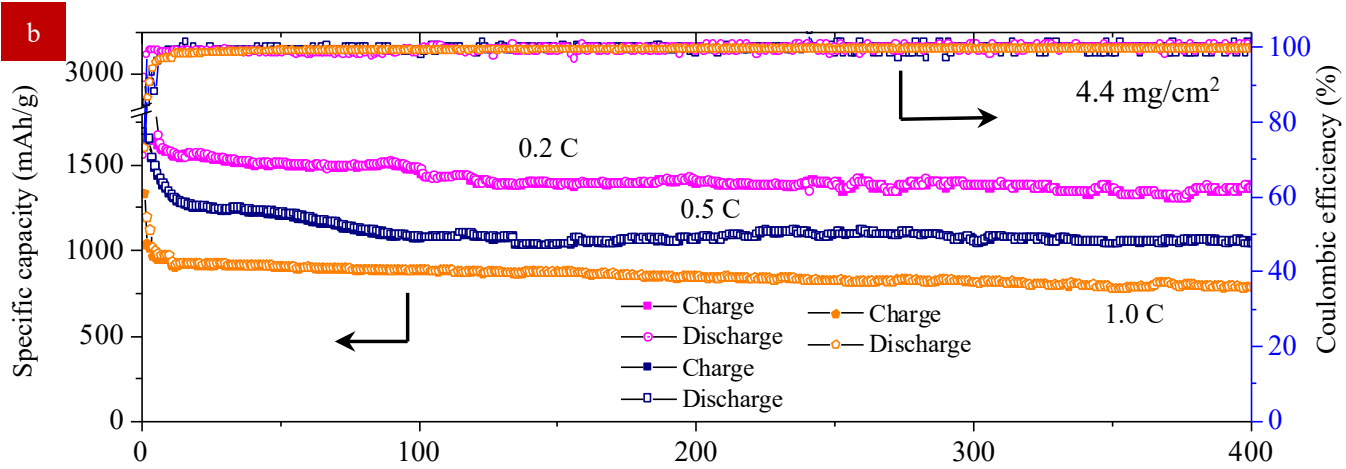

Cycle number

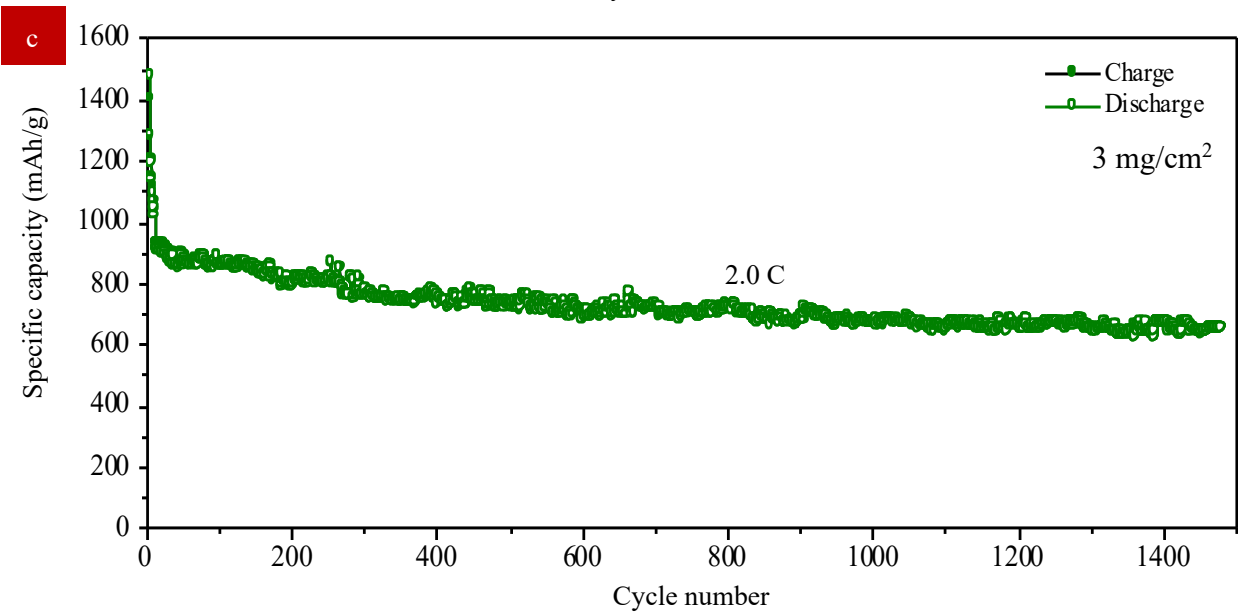

Figure S8. Cycling performance of the battery with $\mu$-Si cage as anode with loadings of $3 \mathrm{mg} / \mathrm{cm}^{2}$ (a, c) and $4.4 \mathrm{mg} / \mathrm{cm}^{2}$ (b) at various rates of $0.2,0.5,1.0$ and $2.0 \mathrm{C}$, respectively. The black curve in (a) is the battery performance with commercial silicon as anode. The Coulombic efficiency was calculated at $0.2 \mathrm{C}$. The specific capacity was calculated based on the overall amount of $\mu$-Si cage (with PPy). 


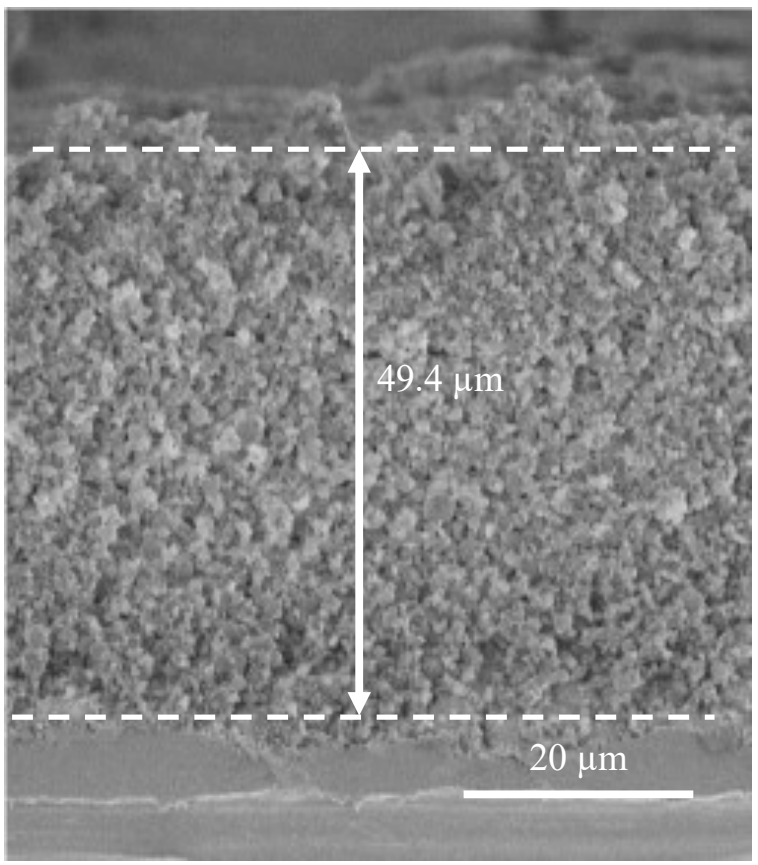

Figure S9. Cross-section SEM image of $\mu$-Si cage coating (the loading is 4.7 $\mathrm{mg} / \mathrm{cm}^{2}$ with an as-received density of $950.2 \mathrm{mg} / \mathrm{cm}^{3}$ ). 


\begin{tabular}{|c|c|}
\hline Samples & Tapped density $\left(\mathrm{g} / \mathrm{cm}^{3}\right)$ \\
\hline$\mu$-Si cage & 0.59 \\
\hline Si nanoparticles $(30 \mathrm{~nm})$ & 0.12 \\
\hline
\end{tabular}
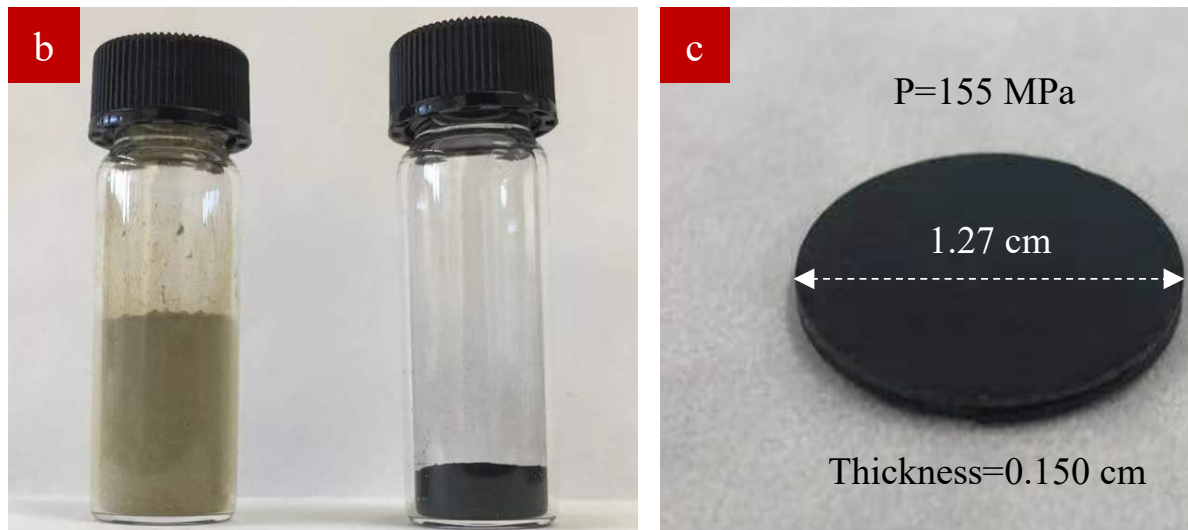

Si NPs $(30 \mathrm{~nm}) \_0.3 \mathrm{~g} \quad \mu$-Si cage_0.3g

Figure S10. (a) Tapped density comparison between the $\mu$-Si cage and 30nm Si nanoparticles (Si NPs). (b) Volume comparison with $0.3 \mathrm{~g}$ sample. (c) Produced pellet under the pressure of $155 \mathrm{Mpa}$ displays a density of $1.32 \mathrm{~g} / \mathrm{cm}^{3}$. 

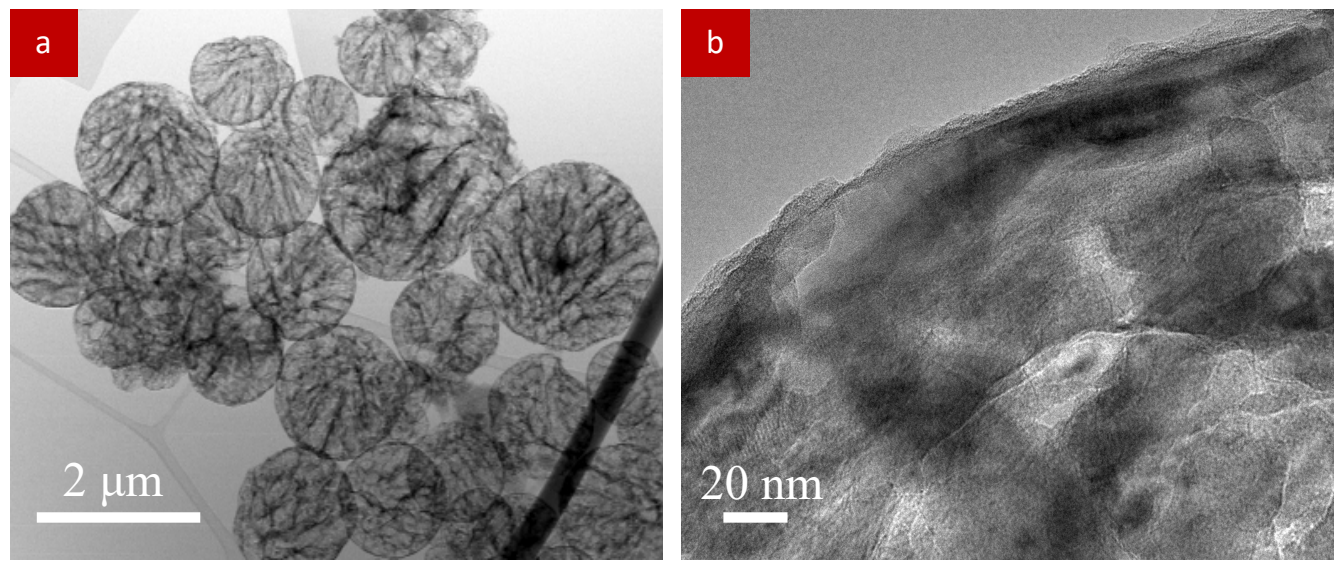

Figure S12. Low-mag (a) and high-mag (b) TEM images of the $\mu$-Si cage. 


\begin{tabular}{|c|c|c|c|c|c|c|c|c|c|c|}
\hline Name & Synthesis method & $\begin{array}{l}\text { Silicon } \\
\text { content } \\
(\%)\end{array}$ & $\begin{array}{l}\text { Volumetri } \\
\text { c capacity } \\
\left(\mathrm{mAh} / \mathrm{cm}^{3}\right)\end{array}$ & $\begin{array}{l}\text { Area } \\
\text { capacity } \\
\left(\mathrm{mAh} / \mathrm{cm}^{2}\right)\end{array}$ & $\begin{array}{l}\text { Specific } \\
\text { capacit } \\
\mathbf{y} \\
(\mathrm{mAh} / \mathbf{g}\end{array}$ & $\begin{array}{l}\text { Current } / \mathbf{r} \\
\text { ate }\end{array}$ & $\begin{array}{l}\text { Cycle } \\
\text { number }\end{array}$ & $\begin{array}{l}\text { First discharge } \\
\text { capacity } \\
(\mathrm{mAh} / \mathrm{g})\end{array}$ & $\begin{array}{l}\text { Loading } \\
\left(\mathrm{mg} / \mathrm{cm}^{2}\right)\end{array}$ & Ref. \\
\hline 3D Nanoporous SiGe Alloy & $\begin{array}{l}\text { Dealloying method from AISiGe } \\
\text { ribbon }\end{array}$ & N/A & $\mathrm{N} / \mathrm{A}$ & $\mathrm{N} / \mathrm{A}$ & 1372 & $100 \mathrm{~mA} / \mathrm{g}$ & 80 & $\sim 3000(100 \mathrm{~mA} / \mathrm{g}$ & $\mathrm{N} / \mathrm{A}$ & 1 \\
\hline $\begin{array}{l}\text { Si nanoparticles } \\
\text { cluster@Si@graphene }\end{array}$ & CVD and wet chemical method & 92 & $\sim 841$ & $\sim 0.76$ & 1388 & $0.5 \mathrm{C}$ & 300 & $3096(0.05 \mathrm{C})$ & 0.6 & 2 \\
\hline \multirow{2}{*}{$\begin{array}{l}\text { Pomegranate design } \mathrm{Si} / \mathrm{C} \\
\text { nanostructure }\end{array}$} & \multirow{2}{*}{$\begin{array}{l}\text { Silicon nanoparticles coated with } \mathrm{SiO}_{2} \\
\text { and carbon }\end{array}$} & \multirow[t]{2}{*}{77} & 1270 & $\mathrm{~N} / \mathrm{A}$ & 1160 & $0.5 \mathrm{C}$ & 1000 & $3,050(0.05 \mathrm{C})$ & 0.2 & \multirow[t]{2}{*}{3} \\
\hline & & & 310 & 3.67 & N/A & N/A & 100 & N/A & 3.12 & \\
\hline Si NP-PANi composited & Polymerization of PANi & 75 & 370.3 & $\sim 0.14$ & 550 & $\sim 2 \mathrm{C}$ & 5000 & $\sim 1900(\sim 0.5 \mathrm{C})$ & $0.2-0.3$ & 4 \\
\hline $\mathrm{Si} / \mathrm{C}$ microsphere & Ball milling and high temperature & 12.5 & $\mathrm{~N} / \mathrm{A}$ & 1.91 & 465 & $0.1 \mathrm{C}$ & 500 & 695 & 4.1 & 5 \\
\hline Amorphous $\mathrm{TiO}_{2} @ \mathrm{Si}$ & Sol-gel coating on commercial Si & 89 & 1032 & 1.068 & 1720 & $0.1 \mathrm{C}$ & 200 & $3061(\mathrm{C} / 30)$ & 0.621 & 6 \\
\hline Porous $\mathrm{Si} / \mathrm{C}$-graphite & $\begin{array}{l}\text { CVD carbon coating on porous } \mathrm{Si} \\
\text { nanoparticles }\end{array}$ & 10 & 750 & 3 & 650 & $\begin{array}{l}0.4 \mathrm{~mA} / \mathrm{cm}^{2} \\
(1 / 6 \mathrm{C})\end{array}$ & 450 & 750 & $\mathrm{~N} / \mathrm{A}$ & 7 \\
\hline Yolk-shell Si@TiO2 & $\begin{array}{l}\text { Commercial Si coated with carbon } \\
\text { and } \mathrm{TiO}_{2} \text {, and chemical etching }\end{array}$ & 73 & $\mathrm{~N} / \mathrm{A}$ & 3 & 990 & $0.05 \mathrm{C}$ & 1500 & 2374 & $0.8-2.1$ & 8 \\
\hline Carbon-coated porous silicon & $\begin{array}{l}\text { Reduction of silica by } \mathrm{Mg} \text {, acid } \\
\text { etching }\end{array}$ & $\sim 82.7$ & $\sim 768$ & 1.0 & 1467 & $0.6 \mathrm{C}$ & 370 & $2445(0.06 \mathrm{C})$ & 1.1 & 9 \\
\hline Nanocage Si-C composite & $\begin{array}{l}\text { Carbon on } \mathrm{SiO}_{2} \text { nanoparticles by } \\
\text { CVD }\end{array}$ & $\mathrm{N} / \mathrm{A}$ & $\mathrm{N} / \mathrm{A}$ & $1.2 \sim 1.5$ & 1433 & $0.5 \mathrm{C}$ & 50 & 1914 & $\mathrm{~N} / \mathrm{A}$ & 10 \\
\hline Ant-nest-like bulk porous silicon & $\begin{array}{l}\mathrm{MgSi} \text { alloy transferred to } \mathrm{Si} \text { and } \\
\mathrm{Mg}_{3} \mathrm{~N}_{2} \text { via nitridation; acid and carbon } \\
\text { coating }\end{array}$ & 91.5 & $\begin{array}{l}1712 \\
(0.1 \mathrm{C})\end{array}$ & $\begin{array}{l}2.8(0.1 \mathrm{~mA} \\
\left.\mathrm{cm}^{-2}\right)\end{array}$ & 1144.3 & $0.5 \mathrm{C}$ & 1000 & $2843(0.05 \mathrm{C})$ & 0.8 & 11 \\
\hline \multirow{6}{*}{$\begin{array}{l}\text { Micro-sized silicon caged by } \\
\text { polypyrrole }\end{array}$} & \multirow[t]{6}{*}{ Wet-chemical method } & \multirow[t]{6}{*}{78.3} & 1410 & 4.3 & 1660 & $0.2 \mathrm{C}$ & 500 & 3664 & \multirow[t]{3}{*}{3.0} & \multirow{6}{*}{$\begin{array}{l}\text { This } \\
\text { work }\end{array}$} \\
\hline & & & 1174 & 3.3 & 1430 & $0.5 \mathrm{C}$ & 500 & 3499 & & \\
\hline & & & 964 & 2.77 & 1149 & $1.0 \mathrm{C}$ & 500 & 3059 & & \\
\hline & & & 1461 & 6.4 & 1660 & $0.2 \mathrm{C}$ & 400 & 3793 & \multirow[t]{3}{*}{4.4} & \\
\hline & & & 1115 & 4.58 & 1328 & $0.5 \mathrm{C}$ & 400 & 3272 & & \\
\hline & & & 848 & 3.48 & 1073 & $1.0 \mathrm{C}$ & 400 & 2329 & & \\
\hline
\end{tabular}

\section{Table S1. Comparison of coin cell battery performance with silicon based materials as anode.}




\section{References:}

1. Yang, Y. H.; Liu, S.; Bian, X. F.; Feng, J. K.; An, Y. L.; Yuan, C. Morphology- and Porosity-Tunable Synthesis of 3D Nanoporous SiGe Alloy as a High-Performance Lithium-Ion Battery Anode. ACS Nano 2018, 12, 2900-2908.

2. Wang, J. Y.; Liao, L.; Li, Y. Z.; Zhao, J.; Shi, F. F.; Yan, K.; Pei, A. L.; Chen, G. X.; Li, G. D.; Lu, Z. Y.; Cui, Y. Shell-Protective Secondary Silicon Nanostructures as Pressure-Resistant High-Volumetric-Capacity Anodes for Lithium-Ion Batteries. Nano Lett. 2018, 18, 7060-7065.

3. Liu, N., Lu, Z., Zhao, J., McDowell, M.T., Lee, H.-W., Zhao, W., and Cui, Y. A Pomegranate-Inspired Nanoscale Design for Large-Volume-Change Lithium Battery Anodes. Nat. Nanotechnol. 2014, 9, 187-192.

4. Wu, H.; Yu, G. H.; Pan, L. J.; Liu, N. A.; McDowell, M. T.; Bao, Z. A.; Cui, Y. Stable Li-Ion Battery Anodes by In-Situ Polymerization of Conducting Hydrogel to Conformally Coat Silicon Nanoparticles. Nat. Commun. 2013, 4, 1943.

5. Xu, Q.; Li, J. Y.; Sun, J. K.; Yin, Y. X.; Wan, L. J.; Guo, Y. G. WatermelonInspired Si/C Microspheres with Hierarchical Buffer Structures for Densely Compacted Lithium-Ion Battery Anodes. Adv. Energy Mater. 2017, 7, 1601481.

6. Yang, J. P.; Wang, Y. X.; Li, W.; Wang, L. J.; Fan, Y. C.; Jiang, W.; Luo, W.; Wang, Y.; Kong, B.; Selomulya, C.; Liu, H. K.; Dou, S. X.; Zhao, D. Y. Amorphous $\mathrm{TiO}_{2}$ Shells: A Vital Elastic Buffering Layer on Silicon Nanoparticles for High-Performance and Safe Lithium Storage. Adv. Mater. 2017, 29 (48), 1700523.

7. Li, X.; Yan, P.; Xiao, X., Woo, J. H.; Wang, C., Liu, J.; Zhang, J. G. Design of Porous $\mathrm{Si} / \mathrm{C}-$ Graphite Electrodes with Long Cycle Stability and Controlled Swelling. Energy Environ. Sci. 2017, 10, 1427-1434.

8. Jin, Y., Li, S., Kushima, A., Zheng, X., Sun, Y., Xie, J., Sun, J., Xue, W., Zhou, G., Wu, J., Shi, F., Zhang, R., Zhu, Z., So, K., Cui, Y.; Li, J. Self-healing SEI Enables Full-Cell Cycling of a Silicon-Majority Anode with a Coulombic Efficiency Exceeding 99.9\%. Energy Environ. Sci. 2017,10, 580-592.

9. Jia, H. P.; Zheng, J. M.; Song, J. H.; Luo, L. L.; Yi, R.; Estevez, L.; Zhao, W. G.; Patel, R.; Li, X. L.; Zhang, J. G. A Novel Approach to Synthesize Micrometer-Sized Porous Silicon as a High Performance Anode for Lithium-Ion Batteries. Nano Energy 2018, 50, 589-597.

10.Son, Y.; Ma, J.; Kim, N.; Lee, T.; Lee, Y.; Sung, J.; Choi, S. H.; Nam, G.; Cho, H.; Yoo, Y.; Cho, J. Quantification of Pseudocapacitive Contribution in NanocageShaped Silicon-Carbon Composite Anode. Adv. Energy Mater. 2019, 9, 1803480. 11.An, W. L.; Gao, B. A.; Mei, S. X.; Xiang, B.; Fu, J. J.; Wang, L.; Zhang, Q. B.; Chu, P. K.; Huo, K. F. Scalable Synthesis of Ant-Nest-Like Bulk Porous Silicon for High-Performance Lithium-Ion Battery Anodes. Nat. Commun. 2019, 10, 1447. 